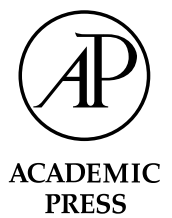

\title{
A priori and a posteriori methods in comparative evolutionary studies of host-parasite associations
}

\author{
Ashley P.G. Dowling, ${ }^{\mathrm{a}, *}$ Marco G.P. van Veller, ${ }^{\mathrm{b}}$ Eric P. Hoberg, ${ }^{\mathrm{c}}$ and Daniel R. Brooks ${ }^{\mathrm{d}}$ \\ ${ }^{a}$ Museum of Zoology and Department of Ecology and Evolutionary Biology, University of Michigan, 1109 Geddes Avenue, Ann Arbor, MI 48109, USA \\ ${ }^{\mathrm{b}}$ Biosystematics Group, Wageningen University, Generaal Foulkesweg 37, 6703 BL Wageningen, The Netherlands \\ ${ }^{\mathrm{c}}$ U.S. National Parasite Collection and the Parasite Biology, Epidemiology and Systematics Laboratory, U.S. Department of Agriculture, \\ Agricultural Research Service, BARC East 1180, 10300 Baltimore Avenue, Beltsville, MD 20705, USA \\ ${ }^{\mathrm{d}}$ Department of Zoology, University of Toronto, M5S 3 G5 Toronto, Ont., Canada
}

Accepted 14 April 2003

\begin{abstract}
Brooks parsimony analysis (BPA) and reconciliation methods in studies of host-parasite associations differ fundamentally, despite using the same null hypothesis. Reconciliation methods may eliminate or modify input data to maximize fit of single parasite clades to a null hypothesis of cospeciation, by invoking different a priori assumptions, including a known host phylogeny. By examining the degree of phylogenetic congruence among multiple parasite clades, using hosts as analogs of taxa but not presuming a host phylogeny or any degree of cospeciation a priori, BPA modifies the null hypothesis of cospeciation if necessary to maintain the integrity of the input data. Two exemplars illustrate critical empirical differences between reconciliation methods and BPA: (1) reconciliation methods rather than BPA may select the incorrect general host cladogram for a set of data from different clades of parasites, (2) BPA rather than reconciliation methods provides the most parsimonious interpretation of all available data, and (3) secondary BPA, proposed in 1990, when applied to data sets in which host-switching produces hosts with reticulate histories, provides the most parsimonious and biologically realistic interpretations of general host cladograms. The extent to which these general host cladograms, based on cospeciation among different parasite clades inhabiting the same hosts, correspond to host phylogeny can be tested, a posteriori, by comparison with a host phylogeny generated from nonparasite data. These observations lead to the conclusion that BPA and reconciliation methods are designed to implement different research programs based on different epistemologies. BPA is an a posteriori method that is designed to assess the host context of parasite speciation events, whereas reconciliation methods are a priori methods that are designed to fit parasite phylogenies to a host phylogeny. Host-switching events are essential for explaining complex histories of host-parasite associations. BPA assumes coevolutionary complexity (historical contingency), relying on parsimony as an a posteriori explanatory tool to summarize complex results, whereas reconciliation methods, which embody formalized assumptions of maximum cospeciation, are based on a priori conceptual parsimony. Modifications of basic reconciliation methods, embodied in TreeMap 1.0 and TreeMap 2.02, represent the addition of weighting schemes in which the researcher specifies allowed departures from cospeciation a priori, with the result that TreeMap results more closely agree with BPA results than do reconciled tree analysis results.
\end{abstract}

(c) 2003 The Willi Hennig Society. Published by Elsevier Science (USA). All rights reserved.

Keywords: Coevolution; A priori methods; A posteriori methods; Brooks parsimony analysis; Reconciled tree analysis; TreeMap; Parsimony; Logical consistency; Phylogenetic systematics; Null hypothesis; Total evidence; Induction; Deduction; Falsification; Verification; Host-parasite associations

Dowling (2002) recently compared the empirical properties of two leading methods used to compare host and parasite phylogenies: Brooks parsimony analysis (BPA; Brooks, 1981, 1985, 1990; Brooks et al., 2001;

\footnotetext{
${ }^{*}$ Corresponding author.

E-mail address: dowlinga@umich.edu (A.P.G. Dowling).
}

Wiley, 1986, 1988a,b) and TreeMap (Page, 1995; now referred to as TreeMap 1.0), based on a series of simulations. His results indicated a preference for BPA over TreeMap 1.0 for two reasons (Dowling, 2002, p. 431): "First, TreeMap grossly overestimates duplications and sorting events when widespread taxa due to hostswitching are present in the associations between the 
host and parasite phylogenies. Second, the ghost taxa that BPA mistakenly produces do not cause any topological changes in the tree, are readily recognizable, and are easy to interpret." These findings were significant, because Dowling's comparisons forced BPA to be assessed using TreeMap criteria. Like the majority of authors who have compared BPA with other methods (e.g., Morrone and Carpenter, 1994; Morrone and Crisci, 1995; Page, 1990a, 1993a; Page and Charleston, 1998; Paterson and Gray, 1997; Paterson and Banks, 2001; Paterson et al., 1999, 2000; Ronquist, 1995, 1996, 1997a,b, 1998; for exceptions, see Hoberg et al., 1997; Van Veller and Brooks, 2001), Dowling (2002) considered only the form of BPA (primary BPA) proposed in 1981 and did not take into account modifications of BPA (secondary BPA), first proposed by Wiley (1986, 1988a,b) and later by Brooks (1990) (see also Brooks and McLennan, 1991, 1993, 2002; Van Veller and Brooks, 2001; Brooks et al., 2001; Green et al., 2002; Hoberg and Klassen, 2002; Brooks and McLennan, 2003). In addition, Dowling's simulations comprised comparisons of only one parasite phylogeny with an a priori specified host phylogeny, whereas BPA is designed to assess cospeciation among multiple parasite clades, in the context of their hosts but without specifying a host phylogeny a priori (Brooks, 1981).

The methods used for phylogenetic analyses of hostparasite associations have been applied to historical biogeographic analyses also. This has been justified by references to analogous concepts and processes between in the of species and areas and in the associations of parasites and hosts (e.g., Brooks, 1981, 1985, 1990; Brooks and McLennan, 1991, 1993, 2002; Page, 1993a, 1995; Page and Charleston, 1998).

In a series of recent studies, Van Veller et al. (1999, $2000,2001,2002)$ investigated some of the empirical properties of different methods of historical biogeographic analysis. They developed a criterion by which the internal consistency of each method could be assessed and were able to identify two categories of methods. They found that each method presented different problems with respect to internal consistency and proposed ways in which those shortcomings could be fixed. In doing this work, Van Veller et al. (2000, 2002, 2003) discovered that the various methods belonged to two distinct classes, which they characterized as a priori and a posteriori. Van Veller and Brooks (2001) suggested that a priori and a posteriori methods represent different research programs stemming from different ontological bases. Ebach and Humphries (2002) strongly disagreed with this position, but their own exemplar and discussion actually supported the position of Van Veller and Brooks (2001) (see Van Veller et al., 2003).

Although Hoberg et al. (1997) noted the contrasts and divergent foundations for BPA and reconciliation methods (implemented in, e.g., component analysis (CA) by Page (1988) and reconciled tree analysis (RTA) by Page (1993b)), they did not explicitly recognize the dichotomy for a posteriori and a priori approaches. Until the study by Dowling (2002) there had been few direct comparisons of these methods, and nearly all had been based on empirical datasets and primary BPA. A contrast was the empirical study of seabirds and tapeworms (Hoberg et al., 1997) that used both secondary BPA and reconciliation methods to assess the history of widespread and sympatric parasite species.

Given that phylogenetic studies of coevolution make use of the same methods, we believe that it is useful to utilize insights recently gained in discussions of historical biogeography in assessment of the controversy about phylogenetic studies of coevolution. We also think that it is important to distinguish between epistemological considerations and ontological considerations to focus attention on the fundamental philosophical differences that have led to the emergence of a priori and a posteriori methods as two very different approaches to the study of host-parasite associations. Epistemologically, our focus is on the application of different methods and the inference and optimization of trees, whereas ontologically, we are concerned with categories of methods, not separate methods. Our over-arching thesis is that a theoretical framework describes a way of obtaining hypotheses from data based upon a researcher's views on the prediction of the relative importance of various processes, in this case, cospeciation, host-switching, duplication, and extinction. The differing opinions on timing, frequency, and likelihood of these coevolutionary processes leads to the dichotomy between a priori and a posteriori methods. We suggest that points of mutual agreement between a priori and a posteriori methods can be viewed as benefiting both research programs. Further, we argue that points of disagreement reflect differences in research programs rather than fundamental flaws in methodology.

\section{Epistemological considerations}

When parasites (of a single clade) are widespread over several hosts or when a single host contains several (sympatric) parasites (of the same clade), there will be only a partial fit between the phylogenies of hosts and those of parasites. It is in these cases that subsequent analyses are necessary to deal with widespread and sympatrically distributed parasites, to infer both common and unique patterns in the associated phylogenies of hosts and parasites. For dealing with widespread and sympatric parasites, among other methods, BPA (Brooks, 1981, 1985, 1990; Brooks et al., 2001; Wiley, 1986, 1988a,b), CA (Nelson and Platnick, 1981; Page, 1988), and RTA (Page, 1993b) can be applied. It has 
generally been assumed that all these methods have been developed to implement the same research program. If this is true, then one way to choose among them is to determine which one best optimizes the goals of the research program. Van Veller et al. (2000) discovered that these different methods (among several others) belong to two different categories based on the different methods that they use for dealing with widespread or sympatric species. In the context of this paper, these widespread or sympatric species are from several clades of parasites distributed over their associated hosts. The distinction between a priori and a posteriori methods is fully discussed in Van Veller et al. (2002). Briefly, a priori methods allow modification of data in the parasite phylogeny to provide maximum fit of a single clade of parasites to a single general host cladogram specified a priori. A posteriori methods do not allow any modification of data in the parasite phylogenies and deal with widespread and sympatric parasites in a parsimony analysis of the unmodified input phylogenies. The most parsimonious depiction of all the data is selected for inferring common patterns in the phylogenies of parasites and hosts that are the result of cospeciation (including sympatric speciation, see below). Parasites whose distributions conflict with these common patterns are explained a posteriori as postspeciation host range expansion (analogous to postspeciation dispersal) or speciation by host-switching (peripheral isolates speciation; see Brooks and McLennan, 1993, 2002). These explanations are obtained by optimizing the data of each clade of parasites on the general host cladogram (primary BPA) or by duplicating the hosts in which the incongruent distributions occur (secondary BPA; for distinctions between primary and secondary BPA, see Brooks et al., 2001; Van Veller and Brooks, 2001; Brooks and McLennan, 2002; Hoberg and Klassen, 2002).

In implementations of reconciliation methods, phylogenetic relationships of widespread and sympatric parasites are reconciled with the host phylogeny by particular combinations of lineage duplication and lineage sorting (e.g., Paterson and Banks, 2001). Initial implementations of these a priori methods did not allow postspeciation host range expansion or speciation by host-switching as possible explanations. TreeMap 1.0 (Page, 1995) and TreeMap 2.02 (Page, 2003) were developed to permit explanations of host switching, but only under conditions determined a priori by the researcher. In the case of TreeMap 1.0, host-switching is determined by first obtaining the maximum number of cospeciations from the initial reconciliation of the two associated phylogenies. From this point, the maximum number of host-switching events is determined (maximum cospeciation minus total number of nodes minus one) and a range from one to the maximum number of host-switches is tried in combination at every node in the phylogeny, searching for any number of host switches that further maximize the total number of cospeciations. TreeMap 2.02 uses the event-based cost analysis model Jungles (Charleston, 1998) to minimize the total cost (host-switching plus duplications plus sorting events), while maximizing the number of cospeciation events.

\section{Putative shortcomings of BPA: two exemplars}

When discussing differences among methods, with the goal of choosing one over the other(s), it is important to make certain that the actual workings of each method are properly represented. Beginning more than a decade ago, Page (1990a, 1994a,b) asserted two major objections to BPA: (1) in some cases BPA may find the same general host cladogram as reconciliation methods, but in doing so may produce biologically unrealistic interpretations of the species involved (variously suggesting too many losses, too many host-switches, or impossible phylogenetic relationships) and (2) BPA will sometimes fail to find the proper general host cladogram (i.e. the host phylogeny). Dowling's (2002) study represented an effort to examine these assertions empirically. The following two exemplars are designed to characterize objections to BPA made by priorists and to further explain the findings by Dowling on purely epistemological grounds.

\section{Exemplar 1}

In Fig. 1a, we show the parasite phylogenies with their hosts superimposed for four clades of parasites. Since no widespread or sympatric parasites are present, no additional steps are necessary and these parasitehost cladograms (in analogy with usage by Kluge, 1998b; Enghoff, 1996) result in resolved host cladograms derived directly from the parasite data congruent with the resolved host cladograms that are obtained for each clade of parasites.

With Component 1.5 (implementation of $\mathrm{CA}$ by Page, 1990b), the sets of resolved host cladograms for different clades are compared for congruent patterns, which are then considered to be the general host cladograms. For this particular exemplar, three of the four clades show a congruent resolved host cladogram, which is considered to be the general host cladogram for these clades (Fig. 1b). The resolved host cladogram for clade T1-T7 that is not congruent with this general host cladogram is ignored and not considered further. Component 2.0 (implementation of RTA by Page, 1993a) selects the same general host cladogram as does Component 1.5. Tree reconciliation for the parasitehost cladograms of three clades (T8-T14, T15-T21, T22-T28) with this general host cladogram needs no 
(a)
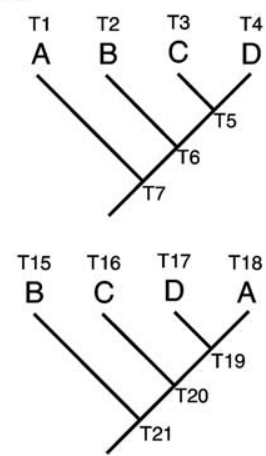
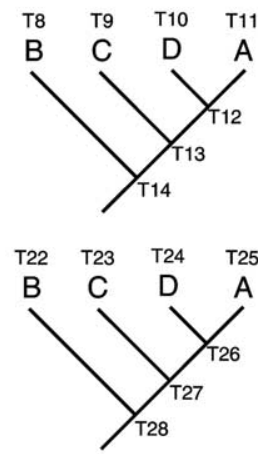

(b)

(c)

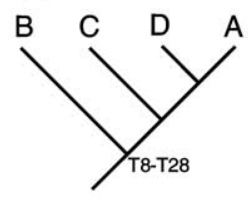

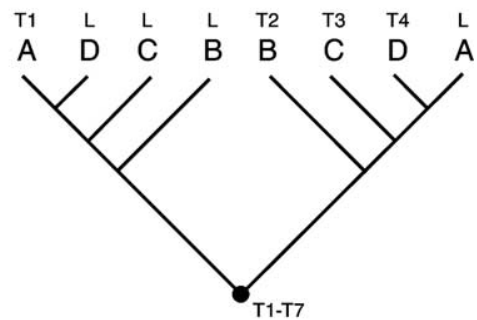

(d)

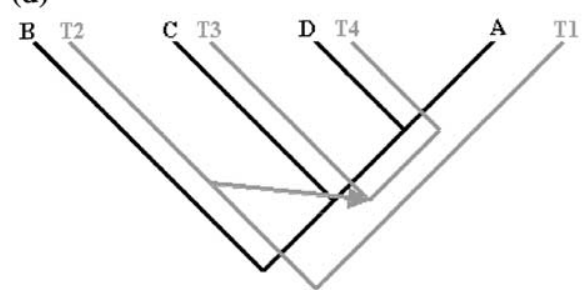

(e)

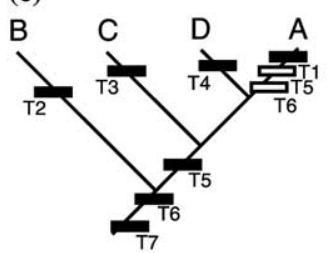

(f)

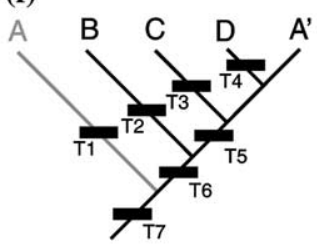

Fig. 1. Exemplar 1 (parasites are represented as T1-28 and the host associations (A-D) are identified at the tips of the clades). (a) Parasite-host cladograms for four clades of parasites; (b) general host cladogram for three of the four clades obtained with Component 1.5; (c) tree reconciliation for the parasite-host cladogram of one clade with the general host cladogram obtained with Component 2.0; (d) reconstruction produced by TreeMap 1.0 using the exhaustive search function and that by TreeMap 2.02 using a default Jungles analysis; (e) general host cladogram for the four clades obtained with primary BPA; (f) general host cladogram for the four clades obtained with secondary BPA); (•, lineage duplication; L, loss; A, B, C, D, hosts; A', host duplication; T1-T28, parasites; $\mathbf{-}$, co-speciation; $\boldsymbol{\bullet}$, loss/host-switch).

duplications or losses. Tree reconciliation for the parasite-host cladogram of the clade consisting of T1-T7 with this general host cladogram needs one lineage duplication and four losses (Fig. 1c).

TreeMap 1.0 and TreeMap 2.02 do not allow the use of multiple parasite phylogenies and, therefore, cannot make an assessment of cospeciation patterns without stipulating a host phylogeny a priori. Due to this limitation of TreeMap 1.0 and TreeMap 2.02, the parasite clades (Fig. 1a) were analyzed individually with a specified host tree, in this case identical to the one chosen by RTA (Fig. 1b). The three parasite clades (T8-T14, T15T21, T22-T28) were perfectly congruent with the host phylogeny and, when analyzed with both TreeMap 1.0 and 2.0, provided results of three cospeciations and no other events. As found with the Component 1.5 and 2.0 analyses, the parasite clade containing $\mathrm{T} 1-\mathrm{T} 7$ is not congruent with the host phylogeny and needs further explanation. TreeMap 1.0 provided an initial reconciliation of one lineage duplication and four losses (the same as the reconciliation done by Components 1.5 and 2.0) and, when host-switching events were exhaustively searched for, a reconstruction involving one host-switch and three losses were recovered (Fig. 1d). This reconstruction requires one less event than the basic reconciliation and therefore is deemed the more desirable reconstruction. Analysis of the same parasite clade (T1-T7) using the Jungles model in TreeMap 2.02, with the default event cost settings (all events equally weighted), recovered the same reconstruction as TreeMap 1.0 (Fig. 1d), with a total cost of four (one host-switch and three losses).

BPA selects the same general host cladogram as do Component 1.5 and Component 2.0 and, therefore, as the a priori host phylogeny for TreeMap. However, the biological interpretation is different. DELTRAN optimization (as preferred by Wiley, 1986, 1988a,b) of the nodes of the parasite-host cladogram of the fourth clade needs nine steps with two homoplasies (Fig. 1e). These homoplasies are explained a posteriori as one loss (reversal) event for parasite T4 and one host-switch (convergence/parallelism) event for parasite T1. In Page's (1990a, 1994a,b) interpretation of BPA, these two events are biologically unrealistic because hypothetical ancestors T5 and T6 (i.e., "ghost taxa" according to Dowling, 2002) arise before parasite T1. One way to deal with this problem is by assuming one persistent ancestor for the hypothetical ancestors $(\mathrm{T} 7=\mathrm{T} 6=\mathrm{T} 5)$. While this interpretation may be appropriate for particular cases, we consider it to be an unsatisfactory means for dealing with the problem.

We believe that the explanation for these biologically unrealistic interpretations is that the data have been forced to conform to an a priori model that they do not support, i.e., to the assumption that hosts cannot have reticulated histories with respect to the parasite species 
that inhabit them. For this particular exemplar the biologically unrealistic interpretations disappear if we assume that host A has a reticulate history. Secondary BPA (Brooks, 1990; see also Brooks and McLennan, 1991, 1993, 2002, 2003; Ruedi, 1996; Brooks et al., 2001; Green et al., 2002; Van Veller and Brooks, 2001) allows a reticulate history for host $\mathrm{A}$ and results in the general host cladogram represented in Fig. If without any biologically unrealistic interpretations.

Comparisons of BPA and reconciliation methods (e.g., Dowling, 2002; Page and Charleston, 1998) have not used secondary BPA. Because parasites may be duplicated in both Component 2.0 (by lineage duplication) and secondary BPA (by host duplication), comparison of the resolved host cladograms obtained with Component 2.0 (RTA) and secondary BPA is more appropriate than comparison of the results obtained with Component 2.0 (RTA) and primary BPA, as has been done previously. This exemplar shows that Component 1.5 , Component 2.0, primary BPA, and secondary BPA all derive the same general host cladogram. BPA provides a more parsimonious explanation of the data than do Component 2.0 (one host-switching event and one loss vs one lineage duplication and four losses) or TreeMap 1.0 and TreeMap 2.0 (one host-switching event and one loss vs one host-switching event and three losses). Secondary BPA also eliminates any apparent unrealistic biological interpretations due to the presence of "ghost lineages" (Dowling, 2002) and eliminates potential controversies arising from the use of DELTRAN or ACCTRAN.

\section{Exemplar 2}

Fig. 2a shows the parasite-host cladograms for three clades of parasites, obtained by replacing the parasites by their hosts. Component 1.5 can deal with the widespread and sympatric parasites in each of the three clades under assumptions 0,1 , and 2 . Under assumption 0 , two of the three clades of parasites show a congruent resolved host cladogram, which is considered to be the general host cladogram for these two clades (Fig. 2b). The only way to obtain a congruent resolved host cladogram for all three clades is by dealing with the widespread and sympatric parasites under assumption 2 . The general host cladogram obtained in the intersection of sets of resolved host cladograms derived under assumption 2 for the three clades is represented in Fig. 2c. Component 2.0 selects the same general host cladogram as is obtained for two of the three clades with Component 1.5 (Fig. 2b) under assumption 0 . Tree reconciliation for the parasite-host cladograms of the three clades with this general host cladogram needs a total of 12 losses and five lineage duplications (Fig. 2d). The general host cladogram that is obtained for the three clades with Component 1.5 under assumption 2 is not selected since tree reconciliation of it with the parasite-host cladograms of the three clades needs 15 losses and six lineage duplications (Fig. 2e). The only way to obtain the general host cladogram that is obtained with Component 1.5 under assumption 2 is by a priori eliminating widespread parasites in favor of endemics (sensu Nelson and Platnick, 1981; Kluge, 1988; Nelson and Ladiges, 1991a,b,c; Page, 1988, 1993a, 1994a).

The three parasite clades (T1-T7, T8-T14, T15-T21) were analyzed individually with TreeMap 1.0 and TreeMap 2.02 using the pectinate host tree (Fig. 2c). TreeMap 1.0 provides an initial reconciliation for parasite clade T1-T7 of two lineage duplications and four losses (Fig. 2f) and an exhaustive search for hostswitching provides no other reconstructions. For parasite clade T8-T14, TreeMap 1.0 provides an initial reconciliation of one lineage duplication and two losses (Fig. 2g), and an exhaustive search for host-switching also provides no other reconstructions. Finally, for parasite clade T15-T21, TreeMap 1.0 produces an initial reconciliation of three lineage duplications and seven losses (Fig. 2h) and, once again, an exhaustive search reveals no other reconstructions. The inability of TreeMap 1.0 to recover host-switching events when dealing with widespread parasites is similar to the results found by Dowling (2002). Since no host-switching events were recovered, TreeMap 1.0 finds virtually the same results as Component. The same analyses using TreeMap 2.02 produce slightly different results. Analysis of parasite clade T1-T7, using TreeMap 2.02, produces two equally good (total cost $=2$ ) reconstructions (Fig. 2i) of one host-switch and one loss but also chooses to completely ignore parasite T2, which should also be associated with host B. Similar results are found when analyzing parasite clade $\mathrm{T} 8$ - T14, from which a reconstruction of perfect congruence to the host phylogeny is recovered (Fig. 2j). Parasite T8, which is associated with hosts A and B, was dropped from host B during the analysis. Finally, for parasite clade T15-T21, the TreeMap 2.02 analysis produced two equally good reconstructions of one host-switch and one loss (Fig. 2k), but this time two widespread parasites were dropped from one of their respective hosts, in this case, T17 was dropped from host $\mathrm{C}$ and $\mathrm{T} 15$ was dropped from host D.

Primary BPA selects the same general host cladogram as does Component 1.5 under assumption 2. DELTRAN optimization of the nodes of the parasite-host cladograms (as suggested by Wiley, 1986, 1988a,b) of the three clades on this general host cladogram needs 25 steps (Fig. 21) with eight homoplasies (four hostswitching events plus associated ancestral codes resulting from inclusive ORing). Secondary BPA, assuming reticulate histories for hosts $\mathrm{A}, \mathrm{B}$, and $\mathrm{D}$, produces the general host cladogram represented in Fig. $2 \mathrm{~m}$, requiring four host-switching events (host duplications) and eliminating the four "ghost lineages." 
(a)

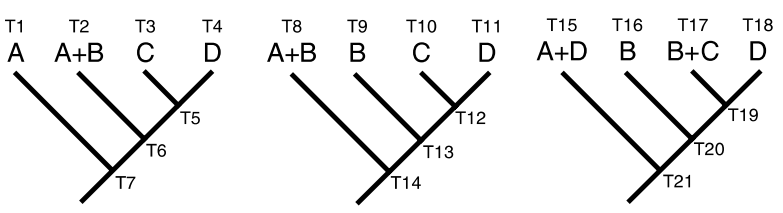

(b)

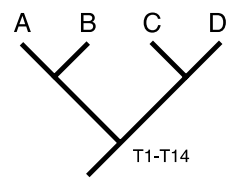

(c)

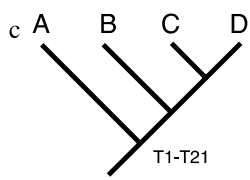

(d)
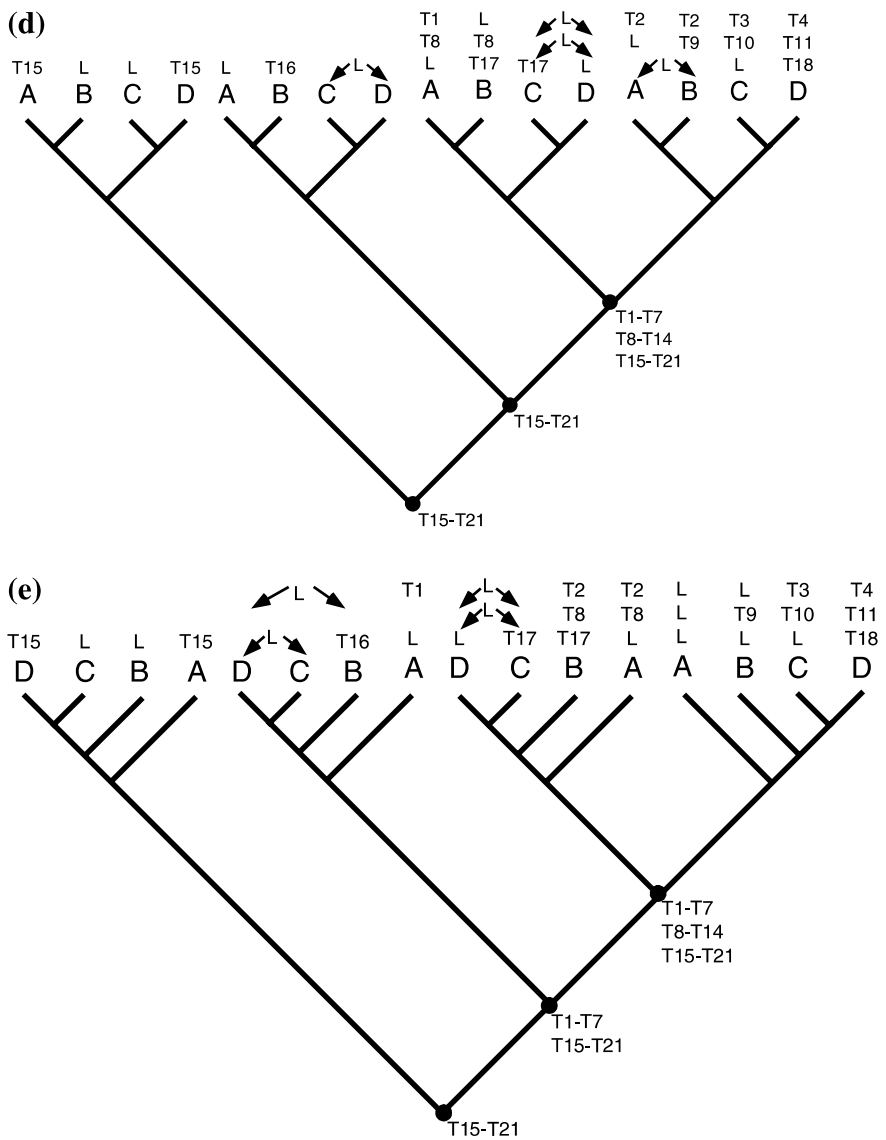

Fig. 2. Exemplar 2 (parasites are represented as T1-28 and the host associations (A-D) are identified at the tips of the clades). (a) Parasite-host cladograms for three clades of parasites; (b) general host cladogram for two of the three clades obtained with Component 1.5 under assumption 0; (c) general host cladogram for the three clades obtained with Component 1.5 under assumption 2; (d) tree reconciliation for the parasite-host cladograms of the three clades with the general host cladogram obtained with Component 2.0; (e) tree reconciliation for the parasite-host cladograms of the three clades with the general host cladogram that is derived by Component 1.5 under assumption 2; (f) host-parasite reconstruction produced by TreeMap 1.0 using the exhaustive search function for parasite clade T1-T7; (g) host-parasite reconstruction produced by TreeMap 1.0 using the exhaustive search function for parasite clade T8-T14; (h) host-parasite reconstruction produced by TreeMap 1.0 using the exhaustive search function for parasite clade T15-T21; (i) two host-parasite reconstructions produced by a Jungles analysis implemented in TreeMap 2.02 for parasite clade T1T7; (j) host-parasite reconstruction produced by a Jungles analysis implemented in TreeMap 2.02 for parasite clade T8-T14; (k) two host-parasite reconstructions produced by a Jungles analysis implemented in TreeMap 2.02 for parasite clade T15-T21; (1) general host cladogram for the three clades obtained with primary BPA; (m) general host cladogram for the three clades obtained with secondary BPA; $(\bullet$, lineage duplication; L, loss; A, B, C, D, host; A', B', B", $\mathrm{D}^{\prime}$, host duplication; T1-T21, parasites; $\boldsymbol{-}$, cospeciation; $\boldsymbol{\square}$, loss/host-switch).

This exemplar shows that the result obtained with primary BPA can be obtained with Component 1.5 and Component 2.0 only by a priori eliminating widespread parasites in favor of endemics, as suggested by Page (1988, 1993a, 1994a) and implemented in TreeMap 1.0 and TreeMap 2.0 (see also Van Veller et al., 2000). When Component 2.0 is forced to use all available data (as BPA does), it chooses a different general host cladogram via tree reconciliation that needs 12 losses and five lineage duplications. To obtain the same general 
(f)

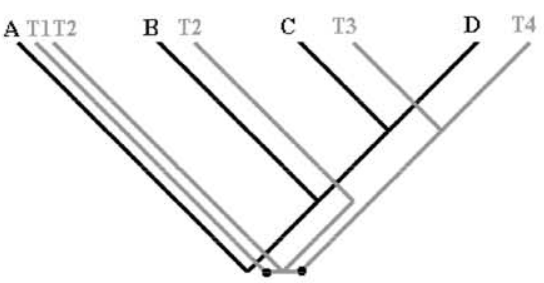

(g)

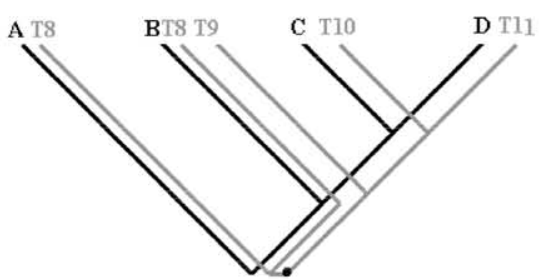

(h)

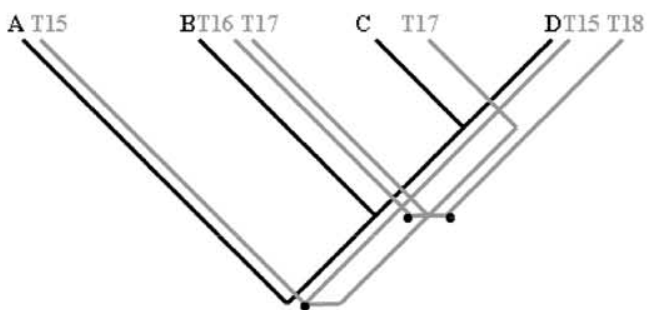

(i)
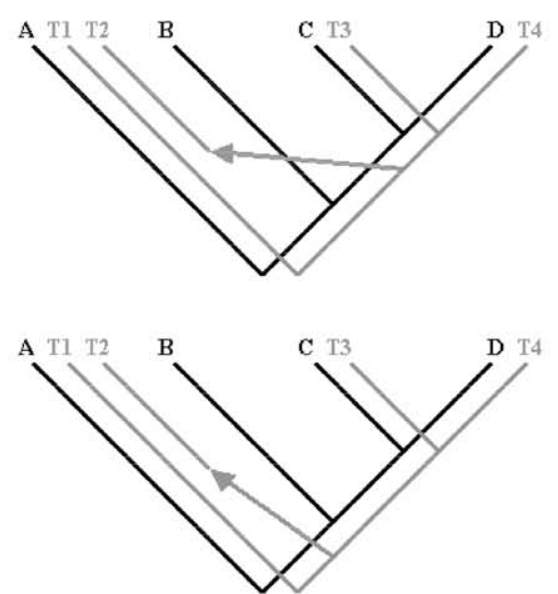

(j)

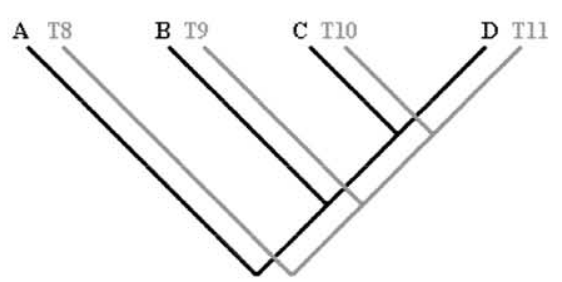

(k)
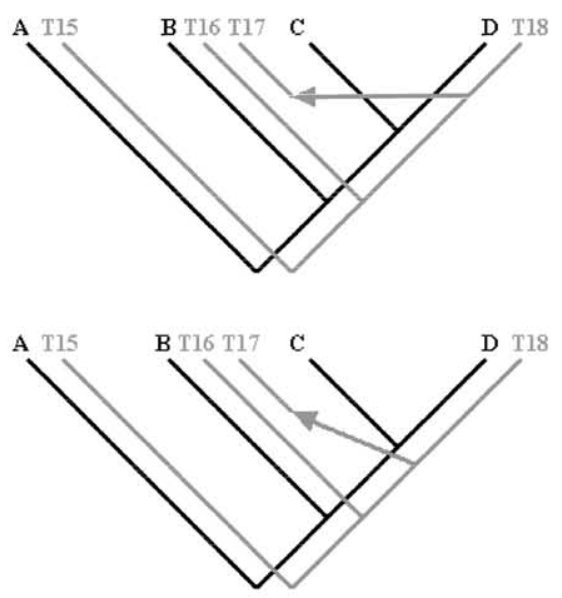

(l)

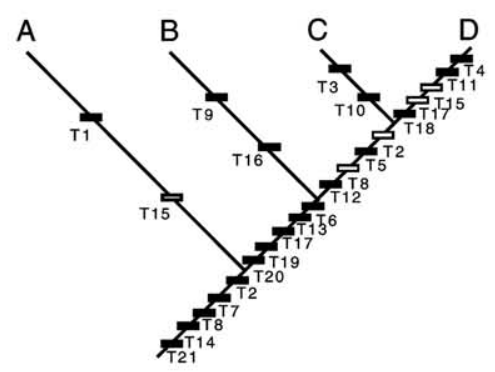

(m)

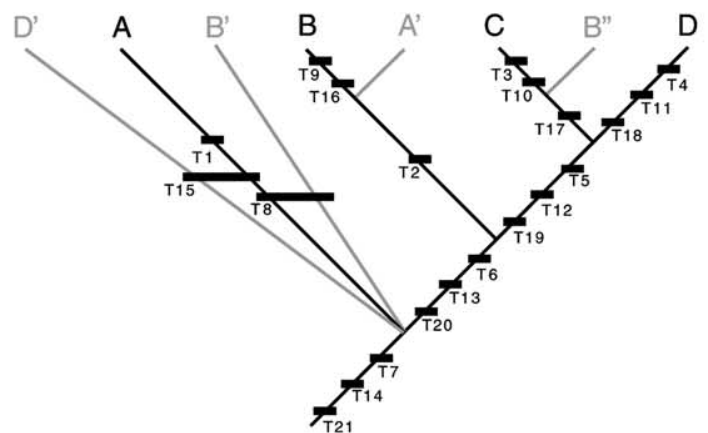

Fig. 2. (continued)

host cladogram that is derived by primary BPA and Component 1.5, Component 2.0 needs 15 losses and six lineage duplications for tree reconciliation, TreeMap 1.0 needs six lineage duplications and 13 losses, and TreeMap 2.02 needs two host-switching events and 2 losses and eliminates two parasite species altogether and half the host records for two other parasite species.

It is thus not primary BPA, but Component 2.0 that does not succeed in finding the general pattern when it is forced to confront all available data from several clades of parasites. Further, secondary BPA explains all data of the three clades in a general pattern with four hostswitching events (host duplication) and no extra steps. Even when reconciliation methods are given the same host tree as that produced by BPA, the BPA result is more parsimonious than the explanation of all data for the three clades with Component 1.0 (five lineage duplications and 12 losses), Component 2.0 and TreeMap 
1.0 (six duplications and 13 losses), or TreeMap 2.02 (two host-switching events and 2 losses, ignoring data from four parasite species).

\section{Discussion}

These two exemplars demonstrate the general circumstances under which various implementations of reconciliation methods produce less parsimonious results than does BPA (see also Ronquist, 1998). This effect is magnified, even to the point of supporting the wrong general host cladogram (exemplar 2), when reconciliation methods are forced to deal with all available data, rather than eliminating some data from the analysis.

The two exemplars show that implementations of reconciliation methods (Component 1.5 and Component 2.0) fail to satisfy two fundamental principles of phylogenetic systematics: using all available evidence (the principle of total evidence: Kluge, 1989, 1997, 1998a,b) and selecting the a posteriori working hypothesis that best fits all available data (the principle of maximum explanatory power: Farris, 1983; see also Kluge and Farris, 1969; Eldredge and Cracraft, 1980; Wiley, 1981; Kluge, 1997, 1998b). Secondary BPA differs from its counterpart, methods that are based on tree reconciliation, with respect to a third and perhaps most important principle of phylogenetic systematics: testability (the principle of falsification: Popper, 1960, 1968a,b, 1972, 1976, 1992; Wiley, 1975; Platnick and Gaffney, 1977, 1978a,b; Gaffney, 1979; Kluge, 1997, 1998b). Invoking subjective a priori weighting schemes, such as those embodied in Jungles, does not correct this shortcoming, although in principle one should always be able to find a weighting scheme under which TreeMap 2.02 will provide the same results as BPA.

Exemplars 1 and 2 demonstrate that when some hosts actually have reticulate histories, primary BPA may produce interpretations that appear to be biologically unrealistic because the data are forced to conform to an a priori all-cospeciation null hypothesis. Those apparently unrealistic interpretations disappear, however, when secondary BPA is performed, allowing the data to falsify the a priori all-cospeciation null hypothesis. BPA, in accordance with the fundamental principles of phylogenetic systematics, functions best as an a posteriori, or falsificationist, method (Popper, 1960, 1968a,b, 1972, 1976, 1992) in conjunction with "cycles of discovery and evaluation" (Frost and Kluge, 1994; Kluge, 1997, $1998 \mathrm{a}, \mathrm{b})$. This is why BPA relies on the a posteriori use of parsimony to explain all the data. Analyses using BPA falsify the null hypothesis of all-cospeciation in each instance that the data warrant such falsification. This is shown visually in secondary BPA, in which each host duplication represents a specific falsification of the null hypothesis of cospeciation.

Reconciliation methods, however, do not attempt to falsify the null hypothesis of cospeciation. Component 1.5 searches for congruent resolved host cladograms in the solution sets obtained for different clades of parasites under the same assumption. Congruent resolved host cladograms, present in the intersection of the solution sets, are selected as general host cladograms and are assumed to verify a common history of cospeciation events by the different clades of parasites. For analyses with Component 1.5 , the chance of finding congruent resolved host cladograms in the intersection of solution sets obtained under assumption 2 is extremely large. General host cladograms with Component 2.0 are derived through tree reconciliation via losses and lineage duplications. The number of resolved host cladograms obtained under assumption 2 with Component 2.0 grows exponentially with an increase in the number of widespread parasites. This is caused by an explosion in the number of a priori rearrangements under assumption 2 (solution sets sensu Van Veller et al., 1999, 2000) to such an extent that the possibility of finding congruent resolved host cladograms that verify a null hypothesis of cospeciation (or any other arbitrarily chosen possibility) approaches certainty. Implementing Jungles does not help, as the realm of potential combinations of a priori "costs" of different coevolutionary phenomena is without limit.

The failure of reconciliation methods to satisfy the three fundamental principles of phylogenetic systematics (mentioned above) corroborate earlier findings in historical biogeography (Van Veller et al., 2000; Van Veller and Brooks, 2001) and coevolution (Dowling, 2002) that when there is disagreement between a priori and a posteriori methods with respect to a general pattern, the result provided by the a posteriori methods is to be preferred. In addition to providing less parsimonious results, it has been shown by Van Veller et al. (2000, 2001) that a priori methods are internally inconsistent on the issue of obtaining inclusive sets of cladograms under assumptions 0,1 , and 2. Advocates of a priori methods, however reject total evidence (Page, 1996), parsimony (Morrone and Carpenter, 1994; Platnick et al., 1996), and inclusive solution sets (Ebach and Humphries, 2002) as criteria for assessing methods and results of a priori and a posteriori approaches. Rather, they prefer to invoke episodes of lineage duplication and lineage sorting of a form and to the extent necessary to increase the apparent support for a single, simple pattern of host relationships (e.g., see Hoberg et al., 1997; Page and Charleston, 1998; Paterson et al., 2000).

To us, such strongly held epistemological views indicate strongly held ontological views. Van Veller and Brooks (2001) suggested that the dispute between advocates of a priori and a posteriori methods has an 
underlying ontological basis. Others have also made this suggestion, either explicitly (Brooks and McLennan, 1991, 1993, 2002, 2003; Ronquist, 1997a,b; Van Veller et al., 2003) or implicitly (Hoberg et al., 1997; Hovenkamp, 1997), but it appears that the perspective needs clarification. Next we consider three categories of distinctions between a priori and a posteriori methods that we believe comprise strongly divergent ontological positions.

\section{Host history versus parasite history perspectives}

We believe that one reason for the marked difference in perspectives represented by the a priori and a posteriori approaches can be traced to fundamental ontological differences of opinion about the nature of parasitism and the evolutionary independence of parasites relative to their hosts. Darwin $(1872$, p. 32) summarized his view of evolution thusly: "there are two factors: namely, the nature of the organism and the nature of the conditions. The former seems to be much more the important, for nearly similar variations sometimes arise under, as far as we can judge, dissimilar conditions; and, on the other hand, dissimilar variations arise under conditions which appear to be nearly uniform." For parasites, hosts are part of the nature of the conditions. Those who consider parasites to be completely dependent on their hosts are not only ignoring half of Darwin's dictum, but are ignoring the half that he considered most important. Methods of analysis based on this ontology are characterized by a set of a priori assumptions used to simplify an analysis; hence, we refer to it as the ontology of simplicity or a priori parsimony (see also Van Veller and Brooks, 2001; Brooks and McLennan, 2002).

To think of parasites as active participants in their own evolution, one must think of parasites as having their own "nature of the organism" (Darwin, 1872). That is, parasites are not simply dependent variables on host phylogeny. Rather, they are independent variables on different host resources, each of which may be distributed in a variety of ways among host species of varying degrees of phylogenetic relatedness (Brooks and McLennan, 1993, 2002). This viewpoint is rooted in (a posteriori methods: Van Veller et al., 2000-2002; Van Veller and Brooks, 2001; Brooks and McLennan, 2002) the same ontology that leads us to believe that we will routinely need to resort to parsimony a posteriori in attempting to study any aspect of the evolution of biodiversity.

Simple versus complex associations between parasites and hosts

When applying a priori methods for studying hostparasite associations one finds the optimal fit of parasite histories to the (a priori determined) host phylogeny and then uses that fit to provide insights into the history of speciation in several unrelated clades of parasites. To implement this maximum cospeciation program, therefore, one must begin with an inferred host phylogeny and one must accept cospeciation as the most probable or usual form of evolutionary association. Within this program the driving assumption is a single origin of all characters or their analogs, thus one parasite for one host or one species for one area; events such as postspeciation host-switching or isolation without speciation are not recognized, though they are real events. Having accepted these assumptions, it is a simple matter of finding the optimal fit of the parasites to the host tree. Maintaining the singularity of host history is crucial for this approach, so it is not surprising that advocates of a priori methods invoke coupled parasite lineage duplication and sorting events rather than host duplication events to deal with mismatches between host and parasite phylogenies.

Studies of host-parasite associations with a posteriori methods may be defined as the search for explanations of the host context of species formation in parasites (see also Wiley, 1986; Brooks and McLennan, 1991, 1993, 2002). Analyses from this (phylogenetic) perspective are based on the assumption that cladograms produced by phylogenetic systematic analysis are hypotheses of speciation events and can thus be used in studies of the evolution of species and of their coevolutionary associations. In addition to (allopatric) cospeciation, processes including sympatric (within-host) speciation, speciation by host-switching (peripheral isolates speciation), postspeciation host-switching, or isolation without speciation (nonresponse to a vicariance event) are also responsible for the occurrence of parasites among hosts (see Brooks and McLennan, 1993; Hoberg, 1995; Hoberg and Klassen, 2002). Distinguishing all these possibilities requires methods that are able to integrate general patterns (host relationships supported by multiple clades of parasites) and unique elements, including those that are incongruent with the general pattern. This is based on the assumption that evolution has been so historically contingent and complex that robust explanations require analysis of both the common and the unique patterns, including the possibility of hosts with reticulated histories relative to the parasites inhabiting them. Representing the unique elements may take the form of optimizing incongruent data onto the general host cladogram (primary BPA) or of duplicating hosts that have a reticulate history with respect to the parasite species inhabiting them (secondary BPA). This evolutionary perspective is ontological rather than epistemological, because if this assumption is not warranted, empirical studies using a posteriori methods will consistently fail to find such complexity and ambiguity, producing results that are congruent with those found 
using a priori methods. A clear example of this point is illustrated by studies of cestodes and seabirds where RTA failed to recover a complex history of hostswitching and diversification (Hoberg, 1992; Hoberg et al., 1997).

\section{Hennig's auxiliary principle versus Hennig's auxiliary principle}

A posteriori methods stem directly from the principles of phylogenetic systematics. In phylogenetic systematics analyses are performed on characters, not taxa (Wiley, 1981; Kluge, 1999). Extending this reasoning to studies of host-parasite associations (from a phylogenetic perspective), a posteriori methods are not analyses of hosts, but analyses of parasites and their relationships in a context of host-associated speciation. It is for this reason that phylogenetic studies of host-parasite associations deal with widespread and sympatric parasites via a parasite-relationship approach rather than a hostrelationship approach. A parasite species cannot have two different histories (hybrid species have two different ancestors, but still only a single history), therefore its occurrence in two different nonsister species hosts must be the result of host-switching unless one parasite species is the persistent ancestor of another (Brooks, 1981, Figs. 39-44; also Johnson and Clayton, 2003). Each such host-switching event produces a reticulated history of the host with respect to the parasite associated with it. These historical reticulations can be depicted as homoplasy in character optimization (primary BPA) or as host duplications (secondary BPA). When applying a priori methods, assumptions 1 and 2 allow parasites or hosts in parasite-host cladograms to be discarded (Nelson and Platnick, 1981; Kluge, 1988; Nelson and Ladiges, 1991a,b,c; Page, 1988, 1993a, 1994a). Ebach (1999) defended this practice by asserting that the discarded taxa are those for which homoplasy is assumed and are not ruled out as required by Hennig's auxiliary principle. The consequence of applying these assumptions in this manner, therefore, is that no falsification of cospeciation among parasites and hosts is possible except those stipulated by the researcher before the data are analyzed. Therefore, a priori methods are inductivist or verificationist in nature. This explains why ambiguity must be treated as "items of error" (Nelson and Platnick, 1981), rather than as "items of falsification" (Van Veller and Brooks, 2001), justifying the use of assumptions 1 and 2 (see also Van Veller et al., 2002). This is the reason that Page and Charleston (1998) can state as fact that BPA underestimates the number of cospeciation events, rather than stating as observation that BPA and reconciliation methods may give different inferences of the numbers of cospeciation events. Ebach and Humphries (2002, p. 430) defined Hennig's auxiliary principle as "homoplasy should not be assumed beyond necessity." If assumptions 1 and 2 are invoked maximally, homoplasy is assumed only to the extent necessary to fit ambiguous data to the general pattern, meaning that the result is as parsimonious as possible given the a priori dictum that Hennig's auxiliary principle cannot be violated. Because a posteriori methods obtain more parsimonious results for data requiring the intervention of assumptions 1 and 2, a priori methods must be assuming homoplasy beyond necessity. Therefore, in treating all ambiguous data as congruence, a priori methods actually violate Hennig's auxiliary principle in an effort to maintain it. This may be a key to understanding why each successive modification of a priori methods has made them more BPA-like (for a chronology of methods developed in comparative studies of host-parasite associations see Table 1).

Phylogeneticists define Hennig's auxiliary principle as "Never presume convergent or parallel evolution; always presume homology in the absence of contrary evidence." (Brooks and McLennan, 2002, p. 36). This principle is not an a priori assumption of a model, but rather a technical presumption used to initiate the analysis; if we assumed that similarity was always due to convergent or parallel evolution, we would never find any evidence of phylogeny. Hennig's auxiliary principle is violated each time that we discover homoplasy (character incongruence), but it is violated a posteriori. This is what makes phylogenetics, and associated a posteriori methods, hypothetico-deductive or falsificationist in nature. Phylogeneticists expect this sort of character incongruence to be a common enough occurrence that one will need to resort to a posteriori invocations of parsimony to choose among competing hypotheses. In studies of host-parasite associations from a phylogenetic perspective, Hennig's auxiliary principle is violated every time that we discover hostswitching events. Homoplasy and host-switching are discovered a posteriori in the context of character congruence, not of a priori character duplication and elimination. a posteriori methods use only assumption 0 precisely to prevent a priori violation of Hennig's auxiliary principle.

Likewise, a posteriori methods assume that no clade of parasites need conform completely to host phylogenetic relationships. Therefore the observation that, under some circumstances, BPA of single parasite clades may not produce a host cladogram congruent with the host phylogeny (noted initially by Brooks $(1979,1981)$ and discussed at length by Brooks and McLennan (1991, 1993, 2002)) indicates not a shortcoming in the methods of analysis, but rather the need to examine more clades of parasites to find host phylogenetic relationships. To the extent that the original basis of BPA is correct, we would expect the results of primary BPA and various a priori methods to converge on the host phylogeny as additional parasite clades are examined. The a priori 
Table 1

Chronology of conceptual and methodological development in comparative studies of host-parasite associations

\begin{tabular}{|c|c|}
\hline Year & Conceptual and methodological development \\
\hline 1966 & $\begin{array}{l}\text { Hennig (pp. 174-180) objects to "the parasitological method" as applied in studies for which only host phylogenetic relationships are } \\
\text { known, with the data from the parasites mapped onto the host phylogeny, as not being robust from a phylogenetic systematic } \\
\text { standpoint. He recommends better elucidation of parasite phyogenies to assess the extent to which parallel phylogenesis of hosts } \\
\text { and parasites is a law (p. 180). }\end{array}$ \\
\hline 1979 & $\begin{array}{l}\text { Brooks demonstrates that, even when phylogenetic relationships of parasites are known, they may exhibit plesiomorphic, } \\
\text { synapomorphic, autapomorphic, or homoplasious relationships with their hosts. He further suggests that the degree of host } \\
\text { specificity is not coupled with the degree of cospeciation. }\end{array}$ \\
\hline 1981 & $\begin{array}{l}\text { Brooks develops BPA and proposes that the obstacles pointed out by Hennig can be overcome if multiple parasite clades are } \\
\text { analyzed simultaneously with respect to their hosts, cospeciation patterns being inferred from phylogenetic congruence among } \\
\text { portions of the parasite phylogenies and host-switching being inferred from incongruence. The expectation is that cospeciation } \\
\text { patterns among parasites will generally conform to host phylogenetic relationships, especially if the parasite clades have very } \\
\text { different transmission biologies. In any event, the host cladogram produced by such multiclade analysis can be tested for } \\
\text { congruence with a host phylogeny generated using data other than parasites. Brooks further suggests that cospeciation between } \\
\text { hosts and parasites will often be the by-product of vicariant speciation affecting host and parasite lineages simultaneously, in } \\
\text { agreement with Hennig (1966, p. 79) who states "Particularly favorable results can be expected if the parasitological method is } \\
\text { supported by geographic vicariance relationships." }\end{array}$ \\
\hline 1983 & Cressey et al. (1983) point out a technical coding problem with BPA, the problem of inclusive ORing, but do not propose a solution. \\
\hline 1985 & Brooks applies BPA in historical biogeography and coevolution, showing fundamental similarities in methods of analysis. \\
\hline $\begin{array}{l}1986, \\
1988\end{array}$ & $\begin{array}{l}\text { Wiley points out a technical problem with BPA resulting from coding absence as plesiomorphic and suggests using "missing data" } \\
\text { coding with a posteriori interpretation of primitive absence vs secondary loss (extinction). }\end{array}$ \\
\hline 1990 & $\begin{array}{l}\text { Brooks proposes the duplication convention (secondary BPA in this paper), which eliminates the problems created by inclusive } \\
\text { ORing. }\end{array}$ \\
\hline 1990 & Page implements component analysis and releases Component 1.5 . \\
\hline 1991 & Brooks and McLennan apply secondary BPA in coevolutionary studies. \\
\hline 1993 & Page implements reconciled tree analysis and releases Component 2.0. \\
\hline $\begin{array}{l}1993, \\
1994\end{array}$ & $\begin{array}{l}\text { Page applies Component } 2.0 \text { in historical biogeography and coevolution, agreeing with Hennig (1966) and Brooks (1985) that both } \\
\text { kinds of research are fundamentally similar. Coevolutionary studies using RTA provide maximal fit of any parasite phylogeny to } \\
\text { an a priori host phylogeny, invoking coupled lineage duplications and extinctions to "reconcile" apparent cases of host-switching } \\
\text { into instances of cospeciation with the host group. }\end{array}$ \\
\hline $\begin{array}{l}1994, \\
1995\end{array}$ & $\begin{array}{l}\text { Page releases TreeMap 1.0. Possible instances of host-switching are allowed by deleting each associate in turn and computing a new } \\
\text { reconciled tree for the remaining associates (Page, 1994a) based on an a priori host phylogeny. In the online manual for TreeMap } \\
\text { 1.0, Page suggests that successive host-switches are allowed (i.e., associate taxa are removed) until the number of putative } \\
\text { cospeciation events stops increasing. }\end{array}$ \\
\hline 1998 & Charleston develops Jungles as an event-based model designed to incorporate host-switching and cospeciation. \\
\hline 2003 & $\begin{array}{l}\text { Page releases TreeMap 2.0. Trial runs involving the same widespread parasites due to host-switching trials that caused problems with } \\
\text { TreeMap } 1.0 \text { in Dowling's (2002) analysis show that while TreeMap } 2.0 \text { (using Jungles) did not produce large numbers of lineage } \\
\text { duplications and extinctions as does RTA and TreeMap 1.0, it did so by deleting the problematic lineage and showing a perfectly } \\
\text { congruent match between the host and the associate phylogeny. }\end{array}$ \\
\hline
\end{tabular}

results, however, would still lack a deductive framework for explaining the incongruences between host and parasite phylogenies, resorting to a priori subjective weighting schemes such as those embodied in various models such as Jungles (see also various contributions in Page, 2003).

\section{Conclusions}

Despite the fact that both a priori and a posteriori methods use the same null hypothesis, they implement different research programs, based on different ontologies (see also Andersson, 1996). A priori methods (CA and RTA) modify the data when necessary to provide maximum fit to the null hypothesis of cospeciation; this amounts to an a priori parsimony criterion. The a priori hypothesis cannot therefore be directly tested or falsified. a posteriori methods (BPA), by contrast, falsify the null hypothesis when the data do not support it; this amounts to an a posteriori parsimony criterion.

The discovery of the difference between these two different approaches permits us to understand the following general properties of a priori and a posteriori methods:

- When cospeciation (including lineage duplications resulting from sympatric speciation) is the most parsimonious explanation for all the data, BPA, CA, and RTA (including TreeMap 1.0 and TreeMap 2.02 as weighted RTA) derive the same resolved host cladograms with the same interpretation (Fig. 3a).

- When losses are the most parsimonious explanation for all the data, BPA, CA, and RTA derive the same resolved host cladograms with the same interpretation (Fig. 3b).

- When losses and host-switching are equally parsimonious explanations for all the data, BPA, CA, and RTA derive the same resolved host cladograms 
(a)
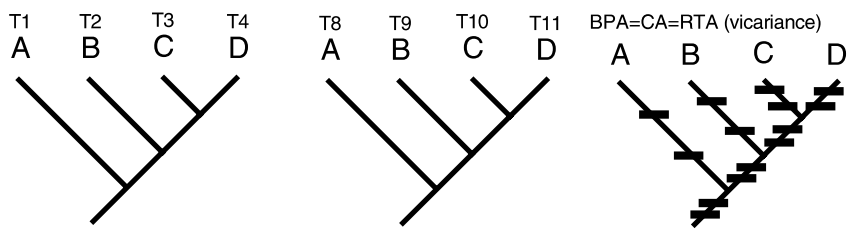

(b)
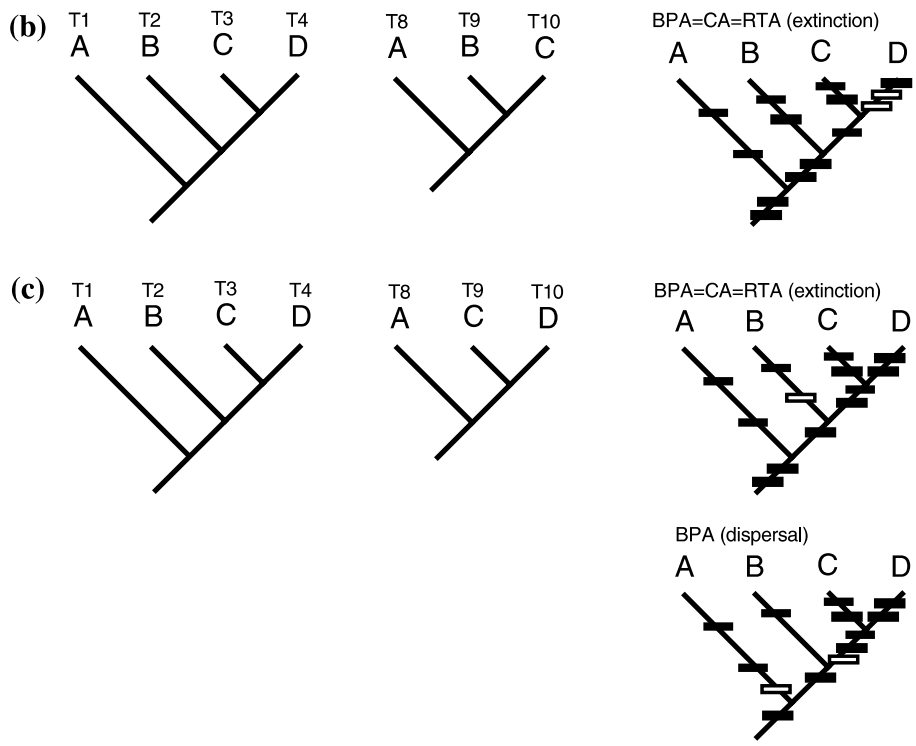

Fig. 3. Similarities and differences in general host cladograms and their interpretations obtained with BPA, CA, and RTA. (a) Cospeciation is most parsimonious explanation; (b) losses are most parsimonious explanation; (c) losses and host-switching are equally parsimonious explanations. (A, B, C, D, hosts; T1-T11, parasites; $\mathbf{-}$, cospeciation; $\boldsymbol{\square}$, loss/host-switch).

(Fig. 3c). However, in interpreting these resolved host cladograms, BPA will choose both losses and hostswitching as equally parsimonious and will require more clades of parasites to resolve the issue, whereas CA and RTA will choose only losses unless an a priori weighting scheme dictates otherwise.

- When host-switching is a more parsimonious explanation for all the data than losses, primary BPA derives the resolved host cladogram based on host-switching (Fig. 21) and secondary BPA depicts each switching event as a falsification of the null hypothesis of cospeciation, signified by the minimal number of host duplications necessary to explain all the switching events (Fig. 2m). CA and RTA, by contrast, either remove data a priori (Fig. 2c) or duplicate parasite lineages and postulate losses a posteriori in sufficient quantity to prevent falsification of the null hypothesis (Fig. 2d). TreeMap 1.0 and TreeMap 2.02 permit departures from maximum cospeciation, but only in a manner and to an extent permitted by an a priori determined weighting scheme.

We think that the above distinctions between a priori and a posteriori methods for comparative studies of host-parasite associations substantiate our claim that they have different ontological bases. Van Veller and Brooks (2001) and Brooks and McLennan (2002) have referred to these as the ontology of simplicity and the ontology of complexity, respectively. If the coevolution of hosts and parasites is predominantly a matter of cospeciation, rather than of historical contingencies, a priori methods are to be preferred. As Hennig (1966) noted, however, we will never know whether cospeciation is common enough to be considered to act in a lawlike manner unless we test the hypothesis using methods that do not presuppose any degree of cospeciation (see also Brooks, 1979, 1981). BPA is such a method, and virtually all coevolutionary studies to date using BPA have discovered significant departures from cospeciation (for references, see Brooks and McLennan, 1993, 2002). Modifications of a priori methods have made them progressively more BPA-like, Despite the modifications, those methods have not achieved the same accuracy in representing all the details of the input data as BPA, nor have they produced a means of assessing cospeciation without a priori acceptance of a host phylogeny and without elimination of inconvenient data. Given our beliefs that phylogenetic relationships among hosts and parasites are complex and that therefore parsimony will be the best that we can do with regard to hypotheses of coevolutionary histories, we are not certain how a priori methods can be suitably modified without reinventing BPA. 


\section{Acknowledgments}

This work was supported by an operating grant from the Natural Sciences and Engineering Research Council of Canada to D.R.B. We express thanks to Rino Zandee, Leiden University, for helpful discussions. We also thank Arnold Kluge, Rod Page, Mark Siddall, and two anonymous referees for their critical and helpful reviews of the manuscript.

\section{References}

Andersson, L., 1996. An ontological dilemma, epistemology and methodology of historical biogeography. J. Biogeogr. 23, 269-277.

Brooks, D.R., 1979. Testing the context and extent of host-parasite coevolution. Syst. Zool. 28, 299-307.

Brooks, D.R., 1981. Hennig's parasitological method: a proposed solution. Syst. Zool. 30, 229-249.

Brooks, D.R., 1985. Historical ecology: a new approach to studying the evolution of ecological associations. Ann. Missouri Bot. Gard. 72, 660-680.

Brooks, D.R., 1990. Parsimony analysis in historical biogeography and coevolution: methodological and theoretical update. Syst. Zool. 39, 14-30.

Brooks, D.R., McLennan, D.A., 1991. Phylogeny, Ecology and Behavior: A Research Program in Comparative Biology. Univ. of Chicago Press, Chicago.

Brooks, D.R., McLennan, D.A., 1993. Parascript: Parasites and the Language of Evolution. Smithsonian Institution Press, Washington, DC.

Brooks, D.R., McLennan, D.A., 2002. The Nature of Diversity: An Evolutionary Voyage of Discovery. Univ. of Chicago Press, Chicago.

Brooks, D.R., McLennan, D.A., 2003. Secondary BPA: extending phylogenetic studies of coevolution. Cladistics.

Brooks, D.R., Van Veller, M.G.P., McLennan, D.A., 2001. How to do BPA, really. J. Biogeogr. 28, 343-358.

Charleston, M.A., 1998. Jungles: a new solution to the host/parasite phylogeny reconciliation problem. Math. Biocsci. 149, 191-223.

Cressey, R.F., Collette, B., Russo, J., 1983. Copepods and scombrid fishes: a study in host-parasite relationships. Fish. Bull. 81, 227265.

Darwin, C., 1872. The Origin of Species, sixth ed John Murray, London.

Dowling, A.P.G., 2002. Testing the accuracy of TreeMap and Brooks parsimony analyses of coevolutionary patterns using artificial associations. Cladistics 18, 416-435.

Ebach, M.C., 1999. Paralogy and the Centre of Origin concept. Cladistics 15, 387-391.

Ebach, M.C., Humphries, C.J., 2002. Cladistic biogeography and the art of discovery. J. Biogeogr. 29, 427-444.

Eldredge, N., Cracraft, J., 1980. Phylogenetic Patterns and the Evolutionary Process. Columbia Univ. Press, New York.

Enghoff, H., 1996. Widespread taxa, sympatry, dispersal, and an algorithm for resolved area cladograms. Cladistics 12, 349-364.

Farris, J.S., 1983. The logical basis of phylogenetic analysis. In: Platnick, N.I., Funk, V.A. (Eds.), Advances in Cladistics II. Columbia Univ. Press, NY.

Frost, D.R., Kluge, A.G., 1994. A consideration of epistemology in systematic biology, with special reference to species. Cladistics 10 , 259-293.

Gaffney, E., 1979. An introduction to the logic of phylogeny reconstruction. In: Cracraft, J., Eldredge, N. (Eds.), Phylogenetic
Analysis and Paleontology. Columbia Univ. Press, New York, pp. 79-111.

Green, M.D., Van Veller, M.G.P., Brooks, D.R., 2002. Assessing modes of speciation: range asymmetry and biogeographical congruence. Cladistics 18, 112-124.

Hennig, W., 1966. Phylogenetic Systematics. Univ. of Illinois Press, Urbana.

Hovenkamp, P., 1997. Vicariance events, not areas, should be used in biogeographic analysis. Cladistics 13, 67-79.

Hoberg, E.P., 1992. Congruent and synchronic patterns in biogeography and speciation among seabirds, pinnipeds and cestodes. J. Parasitol. 78, 601-615.

Hoberg, E.P., 1995. Historical biogeography and modes of speciation across high-latitude seas of the Holarctic: concepts for hostparasite coevolution among Phocini (Pinnipedia) and Tetrabothriidae. Can. J. Zool. 73, 45-57.

Hoberg, E.P., Klassen, G.J., 2002. Revealing the faunal tapestry: coevolution and historical biogeography of hosts and parasites in marine systems. Parasitology 124, S3-S22.

Hoberg, E.P., Brooks, D.R., Siegel-Causey, D., 1997. Host-parasite cospeciation: history, principles and prospects. In: Clayton, D.H., Moore, J. (Eds.), Host-Parasite Evolution: General Principles and Avian Models. Oxford Univ. Press, Oxford, pp. 212-235.

Johnson, K.P., Clayton, D.H., 2003. Coevolutionary history of ecological replicates: comparing phylogenies of wing and body lice to columbiform birds. In: Page, R.D.M. (Ed.), Tangled Trees. Univ. of Chicago Press, Chicago, IL, USA, pp. 262-286.

Kluge, A.G., 1988. Parsimony in vicariance biogeography: A quantitative method and a Greater Antillean example. Syst. Zool. 37, 315-328.

Kluge, A.G., 1989. A concern for evidence and a phylogenetic hypothesis of relationships among Epicrates (Boidae, Serpentes). Syst. Zool. 38, 7-26.

Kluge, A.G., 1997. Testability and the refutation and corroboration of cladistic hypotheses. Cladistics 13, 81-96.

Kluge, A.G., 1998a. Total evidence or taxonomic congruence: cladistics or consensus classification. Cladistics 14, 151-158.

Kluge, A.G., 1998b. Sophisticated falsification and research cycles: consequences for differential character weighting in phylogenetic systematics. Zool. Scr. 26, 349-360.

Kluge, A.G., 1999. The science of phylogenetic systematics: explanation, prediction, and test. Cladistics 15, 429-436.

Kluge, A.G., Farris, J.S., 1969. Quantitative phyletics and the evolution of anurans. Syst. Zool. 18, 1-32.

Morrone, J.J., Carpenter, J.M., 1994. In search of a method for cladistic biogeography: an empirical comparison of Component Analysis, Brooks Parsimony Analysis, and Three-area Statements. Cladistics 10, 99-153.

Morrone, J.J., Crisci, J.V., 1995. Historical Biogeography: introduction to methods. Annu. Rev. Ecol. Syst. 26, 373-401.

Nelson, G., Ladiges, P.Y., 1991a. Standard assumptions for biogeographic analysis. Austral. Syst. Bot. 4, 41-58.

Nelson, G., Ladiges, P.Y., 1991b. Three-area statements: standard assumptions for biogeographic analysis. Syst. Zool. 40, 470-485.

Nelson, G., Ladiges, P.Y., 1991. TAS: Three Area Statements, Program and User's Manual. Published by authors. Melbourne, New York.

Nelson, G., Platnick, N.I., 1981. Systematics and Biogeography: Cladistics and Vicariance. Columbia Univ. Press, New York.

Page, R.D.M., 1988. Quantitative cladistic biogeography: constructing and comparing area cladograms. Syst. Zool. 37, 254-270.

Page, R.D.M., 1990a. Component analysis: a valiant failure? Cladistics 6, 119-136.

Page, R.D.M., 1990b. Component 1.5. Program and User's Manual. Univ. of Auckland, Auckland. 
Page, R.D.M., 1993. Component 2.0: Tree Comparison Software for Microsoft Windows. Program and User's Manual. Natural History Museum, London.

Page, R.D.M., 1993b. Genes, organisms, and areas: the problem of multiple lineages. Syst. Biol. 42, 77-84.

Page, R.D.M., 1994a. Maps between trees and cladistic analysis of historical associations among genes, organisms, and areas. Syst. Biol. 43, 58-77.

Page, R.D.M., 1994b. Parallel phylogenies: reconstructing the history of host-parasite assemblages. Cladistics 10, 155-173.

Page, R.D.M., 1995. Treemap 1.0. Program and User's Manual. Division of Environmental and Evolutionary Biology. Institute of Biomedical and Life Sciences. Univ. of Glasgow, Glasgow.

Page, R.D.M., 1996. On consensus, confidence and "total evidence". Cladistics 12, 83-92.

Page, R.D.M. (Ed.), 2003. Tangled Trees. Univ. of Chicago Press, Chicago.

Page, R.D.M., 2003. Treemap 2.0. Program and User's Manual. Division of Environmental and Evolutionary Biology. Institute of Biomedical and Life Sciences. Univ. of Glasgow, Glasgow.

Page, R.D.M., Charleston, M.A., 1998. Trees within trees: phylogeny and historical associations. Trends Ecol. Evol. 13, 356-359.

Paterson, A.M., Banks, J., 2001. Analytical approaches to measuring cospeciation of host and parasites: through a glass darkly. Int. J. Parasitol. 31, 1012-1022.

Paterson, A.M., Gray, R.D., 1997. Host-parasite co-speciation, host switching and missing the boat. In: Clayton, D.H., Moore, J. (Eds.), Host-Parasite Evolution: General Principles and Avian Models. Oxford Univ. Press, Oxford, pp. 236-250.

Paterson, A.M., Palma, R.L., Gray, R.D., 1999. How frequently do avian lice miss the boat? Implications for coevolutionary studies. Syst. Biol. 48, 214-223.

Paterson, A.M., Wallis, G.P., Wallis, L.J., Gray, R.D., 2000. Seabird louse coevolution: complex histories revealed by $12 \mathrm{~S}$ rDNA sequences and reconciliation analyses. Syst. Biol. 49, 383-399.

Platnick, N.I., Gaffney, E.S., 1977. Systematics: A Popperian perspective. Syst. Zool. 26, 360-365, reviews of "The Logic of Scientific Discovery" and "Conjectures and Refutations" by Karl R. Popper.

Platnick, N.I., Gaffney, E.S., 1978a. Evolutionary Biology: a Popperian perspective. Syst. Zool. 27, 137-141.

Platnick, N.I., Gaffney, E.S., 1978b. Systematics and the Popperian paradigm. Syst. Zool. 27, 381-388.

Platnick, N.I., Humphries, C.J., Nelson, G., Williams, D.M., 1996. Is Farris optimization perfect?: three-taxon statements and multiple branching. Cladistics 12, 243-252.

Popper, K.R., 1960. The Poverty of Historicism. Routledge and Kegan Paul, London.

Popper, K.R., 1968a. The Logic of Scientific Discovery. Harper and Row, New York.

Popper, K.R., 1968b. Conjectures and Refutations. Harper and Row, New York.
Popper, K.R., 1972. Objective Knowledge: An Evolutionary Approach. Clarendon Press, Oxford.

Popper, K.R., 1976. Unended Quest: An Intellectual Autobiography. Open Court Publishing, La Salle, IL.

Popper, K.R., 1992. Realism and the Aim of Science. Routledge, London.

Ronquist, F., 1995. Reconstructing the history of host-parasite associations using generalized parsimony. Cladistics 11, 73-89.

Ronquist, F., 1996. Matrix representation of trees, redundancy, and weighting. Syst. Biol. 45, 247-253.

Ronquist, F., 1997a. Dispersal-vicariance analysis: a new approach to the quantification of historical biogeography. Syst. Biol. 46, 195203

Ronquist, F., 1997b. Phylogenetic approaches in coevolution and biogeography. Zool. Scr. 26, 313-322.

Ronquist, F., 1998. Three-dimensional cost-matrix optimization and maximum co-speciation. Cladistics 14, 167-172.

Ruedi, M., 1996. Phylogenetic evolution and biogeography of Southeast Asian shrews (genus Crocidura: Soricidae). Biol. J. Linn. Soc. 58, 197-219.

Van Veller, M.G.P., Brooks, D.R., 2001. When simplicity is not parsimonious: a priori and a posteriori methods in historical biogeography. J. Biogeogr. 28, 1-11.

Van Veller, M.G.P., Brooks, D.R., Zandee, M., 2003. Cladistic and phylogenetic biogeography: the art and the science of discovery. J. Biogeogr. 30, 319-329.

Van Veller, M.G.P., Kornet, D.J., Zandee, M., 2000. Methods in vicariance biogeography: assessment of the implementations of assumptions zero, 1 and 2. Cladistics 16, 319-345.

Van Veller, M.G.P., Kornet, D.J., Zandee, M., 2002. A posteriori and a priori methodologies for testing hypotheses of causal processes in vicariance biogeography. Cladistics 18, 207-217.

Van Veller, M.G.P., Zandee, M., Kornet, D.J., 1999. Two requirements for obtaining valid common patterns under different assumptions in vicariance biogeography. Cladistics 15, 393-406.

Van Veller, M.G.P., Zandee, M., Kornet, D.J., 2001. Measures for obtaining inclusive solution sets under assumptions zero, 1 and 2 with different methods for vicariance biogeography. Cladistics 17 , 248-259.

Wiley, E.O., 1975. Karl R. Popper, systematics, and classification: a reply to Walter Bock and other evolutionary taxonomists. Syst. Zool. 24, 233-242.

Wiley, E.O., 1981. Phylogenetics: The Theory and Practice of Phylogenetic Systematics. Wiley-Interscience, New York.

Wiley, E.O., 1986. Methods in vicariance biogeography. In: Hovenkamp, P. (Ed.), Systematics and Evolution: A Matter of Diversity. Univ. of Utrecht Press, Utrecht, pp. 283-306.

Wiley, E.O., 1988a. Vicariance biogeography. Annu. Rev. Ecol. Syst. $19,513-542$.

Wiley, E.O., 1988b. Parsimony analysis and vicariance biogeography. Syst. Zool. 37, 271-290. 